\title{
Korruptionsbekämpfung auf Auslandsmärkten durch deutsches Strafrecht?
}

- Gedanken zur gesetzgeberischen Vorgehensweise und zu deren Erfolgsaussichten -*

\section{Einführung}

Korruption wurde - auch von sonst eher nüchternen Zeitgenossen - oft mit sehr drastischen Worten beschrieben: So wurde unter anderem geschrieben, sie sei das „Krebsgeschwür der Welt“, ${ }^{1}$ oder „der Wurm der Apokalypse, der die Demokratie von innen aushöhlt" ${ }^{2}{ }^{2}$ Was treibt unsere Zeitgenossen zu diesen Äußerungen? Dies zu beantworten, fällt nicht sonderlich schwer; um die enorme Schädlichkeit von Korruption besonders plastisch nachvollziehen zu können, bietet sich jedoch ein Blick ins Ausland an. Es ist zwar mitnichten so, dass Deutschland korruptionsfrei wäre. Allerdings erweckt die fast tägliche Presseberichterstattung in der breiten Öffentlichkeit und leider auch bei manchen staatlichen Stellen den Eindruck, es gäbe in Deutschland einen ,ungeheuerlichen Sumpf, der schnellstmöglich trockengelegt werden müsste “. ${ }^{3}$ In jüngster Zeit häufen sich die Nachrichten über Ermittlungsverfahren wegen Korruptionsdelikten in einem Maße, dass man meinen könnte, dass die oft in den Medien geäußerte Einschätzung, Korruption sei in Deutschland eine „Wachstumsbranche“, so verkehrt nicht sein kann. Die Korruptionsverfolgung ist jedenfalls definitiv eine Wachstumsbranche. Gleichwohl ist trotz aller Diskussionen über das korruptionsspezifische Dunkelfeld nicht hinreichend klar, ob und in welchem Maße die Korruption in Deutschland überhaupt zugenommen hat.

Jedenfalls wird wohl die Aussage richtig sein, dass die Bundesrepublik im Vergleich zu vielen anderen Ländern ein doch vergleichsweise noch korruptionsresistenter Staat ist, und dass Bestechungen im Wirtschaftsleben und im Kontakt zu Behörden zwar durchaus vorkommen, jedoch nur in deutlich geringerem Umfang als anderswo. Die Sozialschädlichkeit von Korruption als Phänomen lässt sich in und an dieser Gesellschaft natürlich auch gut studieren; wenn aber die Zerstörungskraft und die spezifische Schädlichkeit von Korruption besonders deutlich zu Tage treten soll, bietet es sich an, seinen Blick nicht nur auf Deutschland zu begrenzen, sondern auch ins Ausland zu blicken.

Als - wirklich abschreckendes - Beispiel kann in diesem Zusammenhang die Russische Föderation dienen. Hier sind Politik, Wirtschaft, Gesellschaft und Justiz in einem

* Der Beitrag basiert auf der Dissertation des Verfassers „Bestechung durch deutsche Unternehmen im Ausland: Strafrechtsentwicklung und Probleme“", die demnächst in den Frankfurter kriminalwissenschaftlichen Studien, Peter Lang-Verlag, Frankfurt am Main, erscheinen wird.

1 So lautet der Titel eines Artikels von Lars Langenau in Spiegel Online vom 20.11.2004, siehe http://www.spiegel.de/politik/deutschland/0,1518,324119,00.html.

2 Dieses Zitat findet sich bei Ostendorf, NJW 1999, S. 615.

3 Siehe dazu Hauschka/Greeve, BB 2007, S. 165. 
für einen westlichen Beobachter kaum für möglich gehaltenen Umfang von Korruption durchsetzt. ${ }^{4}$ Russland gilt als einer der korruptionsanfälligsten Staaten der Welt, vergleichbar mit Staaten wie Sierra Leone, Ruanda, Burundi, Kambodscha oder Turkmenistan. ${ }^{5}$ Die Erfahrung mit Korruption ist „total und existentiell“ und erfasst sämtliche Sphären der Wirtschaft und des täglichen Lebens, sie wird in wissenschaftlichen Studien als endemisches, pervasives und systematisches Phänomen wahrgenommen und für den Bürger als normal, notwendig und alltäglich angesehen. ${ }^{6}$ Teilweise wird deswegen in soziologischen Studien davon ausgegangen, für den modernen Russen sei Korruption „ein vorherrschendes Verhaltensmuster“, wobei die Korruption gegenüber Amtsträgern in den letzten Jahren sogar noch sprunghaft angestiegen ist. Buchstäblich alle Unternehmer sind mit der Möglichkeit, meistens aber mit der Notwendigkeit konfrontiert, Amts- oder Mandatsträger zu bestechen. ${ }^{7}$ Befragungen lassen den Schluss zu, dass zwischen 80 und $90 \%$ der russischen Unternehmer bzw. Manager gelegentlich oder üblicherweise in korruptive Praktiken involviert sind. Dies nicht von ungefähr: Erstens ist nahezu jede Behörde, auch Antikorruptions-, Kontroll- bzw. Aufsichtsbehörden durch Korruption beeinträchtigt, ebenso die Ministerien, ${ }^{8}$ und zweitens muss in der Praxis faktisch für nahezu jede Amtshandlung der Exekutive von Unternehmen bezahlt werden: ${ }^{9}$ Ohne solcherlei Zuwendungen sei eine wirtschaftliche Betätigung faktisch nicht möglich. ${ }^{10}$ Eine unabhängige gerichtliche Kontrolle von Behördenentscheidungen fin-

4 Vgl. statt vieler nur Gilinskij, MSchrKrim 2007 (Heft 6), S. 502 ff. Im Falle Russlands ist allerdings darauf hinzuweisen, dass in der russischen Kriminologiewissenschaft sowie der Devianz- bzw. Sozialforschung unumstritten ist, dass das Korruptionsproblem in Russland in weit stärkerem Maße als in anderen Ländern auf gesellschaftliche Faktoren zurückzuführen ist, deren Vorhandensein geschichtlich weit zurückverfolgt werden kann.

5 Dies ist das Ergebnis des Transparency International Corruption Perception Index (z.B. die Untersuchung aus dem Jahr 2006), siehe dazu auch Stykow, in: Vierteljahreshefte zur Wirtschaftsforschung 73 (2004), S. 247; Russland-Analysen Nr. 120 (2006), S. 15. Weitere Befunde zur Korruption in Russland bei Ledeneva/Kurkchiyan-Varese, Economic Crime in Russia (2000), S. $17 \mathrm{ff}$.

6 Holm, Das korrupte Imperium. Bericht aus Russland (2006), S. 15 ff.; Stykow, in: Vierteljahreshefte zur Wirtschaftsforschung 73 (2004), S. 247 f.; Ledenenva, Russia`s Economy of Favours: Blat, networking and informal Exchange (1998), S. $175 \mathrm{ff}$.

7 Stykow (o. Fn. 5), S. 241 m.w.N.

8 Vgl. die INDEM-Studie „Untersuchung russischer Korruption. Eine soziologische Untersuchung" unter www.anti-corr.ru/awbreport/index.htm. Siehe weiterhin Sungurov, Civil Initiatives and Prevention of Corruption (2000), S. $72 \mathrm{ff}$. Besonders von ,grand corruption“ betroffen sind häufig wirtschaftsnahe Administrationen, z.B. das Finanzministerium, das Wirtschaftsministerium und das Ministerium für Staatsvermögen. Siehe dazu auch Holm (o. Fn. 6), S. 257.

9 Da beispielsweise die Steuerinspektion hochgradig korrumpiert sei, sei es ohne entsprechende Zuwendung nicht möglich, seinen steuerlichen Abschlussbericht abzugeben. Mittlerweile haben sich diverse Marktpreise und „Preislisten“ für unterschiedliche Amtshandlungen bzw. deren Unterlassung gebildet, über die jährlich investigativ von Journalisten berichtet wird. Beispielsweise kostet die Eröffnung eines Restaurants in St. Petersburg aufgrund der legalen Zulassungsprozeduren ca. 30.000 USD. Ungefähr dieselbe Summe muss derzeit an Zuwendungen für verschiedene Amtsträger aufgebracht werden, siehe dazu Holm (o. Fn. 6), S. 18.

10 Gilinskij, MSchrKrim 2007 (Heft 6), S. 502, 506. 
det ebenfalls faktisch nicht statt. Insbesondere die Justiz gilt als in hohem Maße korruptionsgefährdet. ${ }^{11}$

Viel deutlicher kann die Schädlichkeit von Korruption sicher nicht zutage treten. Insofern kann vielen Zeitgenossen beigepflichtet werden, dass sich für einen demokratischen Rechtsstaat erhebliche Gefahren ergeben können, wenn Korruption nicht entschieden bekämpft wird.

\section{Gesetzgeberische Aktivitäten}

Eine derartige Ausbreitung von Korruption wie soeben beschrieben mag dabei auch für die Bundesrepublik eine Motivation gewesen sein, die bestehende Rechtslage zu modifizieren. Früher ,versagte“ das deutsche Strafrecht bei Korruption im Ausland häufig, weil es wegen der alten Definition des (deutschen) Amtsträgers und wegen den Restriktionen der $\S \S 3 \mathrm{ff}$. StGB nicht zur Anwendung gebracht werden konnte; dies ist jetzt anders. Der deutsche Gesetzgeber hat sich entschieden, nicht nur in Frankfurter Baubehörden oder Kölner Entsorgungsbetrieben gegen Korruption vorzugehen, sondern auch bei Korruption, die im Ausland stattfindet, nunmehr energisch durchzugreifen.

Der Anstoß zu den deutschen Aktivitäten kam allerdings mittelbar aus den USA. Diese hatten bereits 1977 den Foreign Corrupt Practices Act (FCPA) ${ }^{12}$ verabschiedet, ein Gesetz, das amerikanischen Unternehmen unilateral die Bestechung ausländischer Amtsträger und Politiker verbat. Allerdings wurde die Wirksamkeit des FCPA durch Kritiker bereits früh bestritten, da amerikanische Firmen auch weiterhin im Ausland Schmiergelder in erheblichem Umfang zahlen würden. ${ }^{13}$ Gleichwohl wurde das Gesetz mit Rücksicht auf die harsche Kritik des amerikanischen Schrifttums sowie der betroffenen amerikanischen Exportwirtschaft, die sich im Vergleich zur ausländischen Konkurrenz benachteiligt sah, im Jahre 1988 revidiert und erheblich entschärft. Zudem versuchten die Vereinigten Staaten, auf Ebene der OECD Druck mit dem Ziel auszuüben, dass sich auch andere OECD-Mitglieder bereiterklären, ähnliche Regelungen einzuführen.

Dies geschah 1997, als es zur Unterzeichnung einer OECD-Konvention über die Bekämpfung der Bestechung ausländischer Amtsträger im Internationalen Geschäftsverkehr kam. ${ }^{14}$ Das hierauf bezugnehmende Umsetzungsgesetz der Bundesrepublik Deutschland (Gesetz zur Bekämpfung internationaler Bestechung - IntBestG) ${ }^{15}$ trat am 15.2.1999 in Kraft und bezweckt den Schutz offener und wettbewerblich strukturierter

11 Salagev/Shashkin/Konnov, in: The Journal of Power Institutions in Post-Soviet Societies (4/5) (2006), http://www. pipps.org/document449.html; Russland-Analysen 120/06-Schmidt, S. 6, 12; Holm (o. Fn. 6), S. 21. Bemerkenswert ist, dass der jetzige russische Präsident Dmitrij Medwedew als Präsidentschaftskandidat während einer Rede am 15.2.2008 wörtlich auf Gerichtsurteile aufgrund von Telefonanrufen ,von oben“ (po zvonku) sowie auf Gerichtsurteile „gegen Geld“ (po dengam) eingegangen ist. Ein auszugsweiser Abdruck der Rede findet sich in den Mitteilungen Nr. 36-37 (Jg. 19/2008) der Vereinigung für deutsch-russisches Wirtschaftsrecht, S. $52 \mathrm{ff}$.

1215 US Code Sect. 78 a, a, m, $78 d d-1$ ff., 78 f.; (geändert 1988 und 1998), zu finden unter www.usdoj.gov/criminal/fraud/fcpa/dojdocb.htm.

13 Vgl. nur Heimann, Entwicklung und Zusammenarbeit 1994, S. 329.

14 Siehe http://www.oecd.org/dataoecd/46/37/35067365.htm.

15 Abgedruckt bei Fischer, StGB, im Anhang 22. 
Märkte vor den negativen Auswirkungen der Korruption. Um den Schutz des weltweiten korruptionsfreien Wettbewerbs als Ziel realisieren zu können, wurde demgemäß die Bekämpfung der Bestechung ausländischer Amtsträger im internationalen Rechtsverkehr mit den Mitteln des deutschen Strafrechts im IntBestG vorgesehen. ${ }^{16}$ Damit wurden dringend für regulierungsbedürftig gehaltene Lücken bei der Strafbarkeit der grenzüberschreitenden Bestechung und der Anwendbarkeit des deutschen Strafrechts auf Auslandssachverhalte geschlossen.

Kern-, aber nicht alleinige Regelung des IntBestG ist die Ausdehnung des Amtsträgerbegriffs für den Anwendungsbereich der aktiven Bestechung i.S.d. § 334 StGB. Dieser umfasst nunmehr nicht nur nach deutschem Recht verpflichtete Amtsträger, sondern auch Amtsträger und Richter ausländischer Staaten bzw. internationaler Organisationen. Weiterhin wurde ein Straftatbestand geschaffen, der die Bestechung ausländischer Abgeordneter im Zusammenhang mit dem internationalen Wirtschaftsverkehr regelt. ${ }^{17} \mathrm{Um}$ somit die unter Strafe gestellten Verhaltensweisen erschließen zu können, ist das StGB mit dem IntBestG zusammenzulesen; hieraus ergibt sich somit folgender „Strafbefehl“:

„Wer, um sich oder einen Dritten einen Auftrag oder einen unbilligen Vorteil im internationalen Geschäftsverkehr zu verschaffen oder zu sichern, einem Amtsträger, Richter oder einen Soldaten im Sinne des $\S 1$ IntBestG einen Vorteil für diesen oder einen Dritten als Gegenleistung dafür anbietet, verspricht oder gewährt, dass er eine richterliche oder sonstige Diensthandlung künftig vornehme und dadurch seine Dienstpflichten verletzen würde, wird bestraft.“

Interessant hierbei ist, dass die deutsche Strafgewalt nicht auf das Gebiet der OECDUnterzeichnerstaaten begrenzt bleibt, sondern auf der ganzen Welt gilt, auch wenn der betroffene Staat die OECD-Konvention gar nicht unterzeichnet hat.

Zusätzlich wurde vom deutschen Gesetzgeber das EUBestG ${ }^{18}$ verabschiedet. Dabei handelt es sich um die deutsche Umsetzung europarechtlicher Vorgaben. Vorausgegangen war die Bereitschaft der Bundesrepublik Deutschland, aufgrund des fortgeschrittenen Integrationsprozesses auf europäischer Ebene die Einbeziehung von europäischen Amtsträgern in den Schutzbereich der Amtsdelikte vornehmen zu wollen. ${ }^{19} \mathrm{Ge}-$ mäß Art. 2 § 1 I EUBestG wird der Anwendungsbereich des $\S 11$ I Nr. 2, 3 StGB im Tatbestandsbereich der $\S \S 332,334-336,338$ StGB, also der aktiven und passiven Bestechung für eine öffentlich-rechtliche Diensthandlung oder eine richterliche Handlung erweitert. Einem deutschen Amtsträger steht somit nach Art. 2 § 1 I EUBestG ein Amtsträger eines anderen EU-Staates, ein Gemeinschaftsbeamter sowie ein Mitglied der Europäischen Kommission und des Rechnungshofes der EG gleich, und einem deutschen Richter steht nunmehr ein Richter eines anderen EU-Mitgliedstaates oder eines Gerichts der EG gleich. Diese Gleichstellung gilt jedoch nur für künftige und pflichtwidrige Dienst- oder richterliche Handlungen.

Somit hat die Bundesrepublik die in diesem Bereich ${ }^{20}$ von ihr für strafwürdig erachteten Lücken geschlossen. Deutsche Staatsanwälte können nunmehr deutsches Straf-

16 Borchmann NJW 2000, S. 254, 260.

17 Wentzell, Zur Tatbestandsproblematik der $\S \S 331,332$ StGB unter besonderer Berücksichtigung des Drittvorteils (2004), S. $100 \mathrm{ff}$.

18 Abdruck bei Fischer, Anhang 21.

19 Siehe dazu Möhrenschlager, JZ 1996, S. 822, 830.

$20 \S 299$ III StGB soll in diesem Beitrag außen vor bleiben. 
recht also nicht nur in Köln oder Stuttgart, sondern auch in Griechenland, Sierra-Leone oder Turkmenistan zur Anwendung bringen.

Bei der Tatbestandsausgestaltung sind dem deutschen Gesetzgeber zumindest im Hinblick auf das IntBestG jedoch erhebliche Versäumnisse vorzuwerfen, da es aufgrund der völlig unbestimmten Tatbestandsmerkmale, die im Gesetz selbst oder in dessen Begründung unverständlicherweise nicht einmal ansatzweise konkretisiert worden sind (was bei der Schwierigkeit der Materie jedoch angezeigt gewesen wäre), ${ }^{21} \mathrm{zu}$ erheblichen Anwendungsproblemen kommt. Insbesondere die Amtsträgerdefinition, welche ,autonom“ im Sinne der OECD-Konvention auszulegen ist, ${ }^{22}$ aber auch die unbestimmten Tatbestandsmerkmale „Öffentliches Unternehmen“, „öffentliche Aufgaben“ oder beauftragte Person im Sinne des Art. $2 \S 1$ Nr. 2 IntBestG sind hierbei misslungen. Insgesamt kommt es bei der Normanwendung zu erheblichen und kaum mehr erträglichen Rechtsunsicherheiten, ${ }^{23}$ wofür der Gesetzgeber zu kritisieren ist. Die vielfältigen Schwierigkeiten der Tatbestandsausgestaltung der Norm sowie deren Rechtsanwendung sollen hier nicht weiter erörtert werden. ${ }^{24}$ Im Rahmen dieses Beitrages soll daher der Befund genügen, dass es erklärtes Ziel der OECD gewesen ist, eine möglichst weite Bandbreite von Lebenssachverhalten von Bestechungen zugunsten der „Foreign Public Officials" erfassen zu können. Der bundesdeutsche Gesetzgeber hat die aus der entsprechenden Umsetzung resultierende Rechtsunsicherheit bewusst in Kauf genommen. Es wurde nicht nur die Weite und Unbestimmtheit der Tatbestandsmerkmale für das deutsche Strafrecht übernommen, sondern zusätzlich noch unterlassen, nähere Anhaltspunkte für die Auslegung des Amtsträgerbegriffs - wenigstens in den Gesetzesmaterialien - zu liefern. ${ }^{25}$

\section{Der internationale Wettbewerb als Schutzgut des Intbest $G$ und dessen Rückwirkungen auf das Schutzgut der $\$ \S 331 \mathrm{ff}$. StGB}

Weiteren Problemen begegnet man, wenn man versucht, sich dem Schutzgut des IntBestG zu nähern.

Die Formulierungen des IntBestG und des OECD-Abkommens legen den Schluss nahe, dass mit dem IntBestG als deutsche Transformation der OECD-Vorgaben nicht primär die Lauterkeit der ausländischen Verwaltung, sondern die Lauterkeit des internationalen Wettbewerbs geschützt werden sollte. Jedenfalls offiziell sollte die Integrität

21 Denn immerhin geht es nicht nur um reine Inlandssachverhalte, deren Handhabung für den Rechtsanwender schon schwierig und unübersichtlich genug ist, wie beispielsweise die sich ständig fortentwickelnde Judikatur und Diskussion zum deutschen Amtsträgerbegriff zeigt. Vielmehr geht es um Auslandssachverhalte und somit um Zuwendungsempfänger, die auch bei primär ,,autonomer“ Tatbestandsauslegung gemäß der OECD-Konvention in erheblichem Maße zumindest auch nach ausländischem Recht zu beurteilen sind; siehe dazu auch MüKoKorte, $\S 334$ Rn. 7 Fn. 8.

22 Vgl. nur Satzger, NStZ 2009, S. 297, 304.

23 Vgl. nur Tinkl, wistra 2006, S. 126 ff.

24 Für eine ausführliche Untersuchung der einzelnen Tatbestandsmerkmale siehe Horrer, Bestechung durch deutsche Unternehmen im Ausland (2010), etwa 2. Kapitel B I, II ff. sowie Tinkl, wistra 2006, S. $126 \mathrm{ff}$.

25 Horrer (o. Fn. 24), S. 246 ff. 
der ausländischen Verwaltungsapparate nicht das ,primäre“ Schutzgut darstellen. Dies hätte auch zu unauflösbaren Konflikten mit der Souveränität anderer Staaten geführt, da die Organisation administrativer Strukturen zum Kernbereich staatlicher Machtausübung und Regelungskompetenz gehört.

Damit ist allerdings noch nicht geklärt, wie das Schutzgut konkretisiert werden könnte. Bei der näheren Bestimmung des Schutzgehalts treten hier erhebliche Schwierigkeiten zutage, da unklar ist, ob das IntBestG deutsche oder alternativ ausländische Staats- oder Individualinteressen an einem lauteren Weltmarkt schützen will oder ob die Institution des Weltmarkts als kollektives Gut der Staatengemeinschaft an sich geschützt werden sollte. Da nach der Gesetzesbegründung ${ }^{26}$ zum IntBestG der Schutz offener und wettbewerblich strukturierter Märkte vor Korruption intendiert war, scheint es jedenfalls um irgendeine Art sozial-überindividueller Rechtsgüter zu gehen. Eine einheitliche, präzise Sichtweise bezogen auf den Schutzgehalt liegt bisher nicht vor; so ist das Schutzgut lediglich in den sehr relativen und schwer fassbaren Kategorien der „Wirtschaftsordnung“, des inländischen „Finanzplatzes“, der „wirtschaftlichen Entwicklung“ und der ,internationalen Wettbewerbsbedingungen“" gesehen worden. ${ }^{27}$

Als Zwischenergebnis bleibt jedenfalls festzuhalten: Das Schutzgut des IntBestG, welches ja auf die deutschen Bestechungsverbote im StGB verweist, ist zumindest nicht mehr nur gleichzusetzen mit den Schutzgütern der Bestechungsdelikte nach dem StGB, sondern geht über diese hinaus. Denn das Schutzobjekt der Lauterkeit der nationalen Verwaltung bzw. des darin gesetzten Vertrauens kann im Hinblick auf das IntBestG nicht (mehr) genügen; vielmehr wird man durch die Einfügung des IntBestG in das deutsche Normensystem als erweitertes Schutzgut der StGB-Bestechungsdelikte auch die Freiheit und Lauterkeit des internationalen Wettbewerbs ansehen müssen. ${ }^{28}$ Die Umsetzung des OECD-Abkommens hat also nichts anderes zur Folge als eine Sprengung der Grenzen des bisherigen Rechtsguts der $\S 331$ ff. StGB. ${ }^{29}$

Der internationale Wettbewerb wirft aber weitere Probleme vor allem grundsätzlicher Art im Hinblick auf sein Dasein als Schutzobjekt strafrechtlicher Normen auf. Bereits der „freie Wettbewerb“ und der „faire Wettbewerb“ sind relative Kategorien, die sich als Schutzobjekt zumindest für das Strafrecht schlecht eignen. ${ }^{30}$ Weitergehend könnte man mit den überzeugenden Argumenten von Albrecht ${ }^{31}$ sogar grundsätzlich anzweifeln, ob das Rechtssystem und das Wirtschaftssystem überhaupt systemisch kompatibel sein können. Zudem ist - nicht nur für Anhänger des Kernstrafrechts - Skepsis gegenüber dem Rechtsgutsbegriff jedenfalls insoweit angebracht, als der internationale Wettbewerb nicht ohne Weiteres eine vom Strafrecht zu schützende „Autonomiezone“ der

26 BTDrs. 13/10428, S. 1.

27 Zusammenstellung bei Androulakis, Die Globalisierung der Korruptionsbekämpfung (2007), S. 398, teilw. auch bezogen auf die zugrundeliegende OECD-Konvention und das Wettbewerbsstrafrecht.

28 Siehe Überhofen, Korruption und Bestechungsdelikte im staatlichen Bereich (1999), S. 234; Randt, BB 2000, S. 1006, 1008; Hild, StraFo 2000, S. 221; Tinkl, wistra 2006, S. 126, 127; Greeve/Dörr in Volk, Münchener Anwaltshandbuch, S. 914 (§ 19 Rn. 244); Weigend, in: FS Jakobs, S. $747 \mathrm{ff}$.

29 Horrer (o. Fn. 24), S. 225 ff., 234 ff.; Fischer, § 331 Rn. 3, wo allerdings auf den faktisch erreichten Schutz der Verwaltung abgestellt wird.

30 Lüderssen, BB 1996, Beilage 11 H.25, S. 8; Albrecht, in: FS Hamm, S. 1, 13 ff.

31 Vgl. dazu u.a. Albrecht, in: FS Hamm, S. 1, 13 ff. 
Rechtsunterworfenen darstellen kann, die sich klar umreißen ließe. Das Strafrecht dürfte angesichts dieses vorgegebenen Rechtsgutsbegriffs jedenfalls deutlich überfordert sein, wenn es Systemschutz, und das sogar noch weltweit, betreiben wollte (siehe dazu sogleich).

Wenn aber ein Interesse an der Lauterkeit des internationalen Wettbewerbs mit den Werkzeugen eines jetzt weltweit geltenden deutschen Strafanspruchs geschützt werden soll, muss zuvor die Frage geklärt werden, ob es ein weltweit zu schützendes gemeinsames Interesse der Staatengemeinschaft an der Reinheit des Weltmarktes überhaupt gibt. Es dürfte heutzutage Allgemeingut sein, dass in vielen Staaten ohne (kleinere) Bestechungsgelder durch die lokalen Administrationen nicht einmal ein Mindestmaß an gewerblicher Tätigkeit ermöglicht wird. ${ }^{32}$ Oftmals werden Schmiergelder gerade in denjenigen Ländern von den Beamten aktiv und als selbstverständlich eingefordert, die über komplizierte bzw. hochbürokratisierte und schon deswegen korruptionsanfällige Verwaltungsverfahren verfügen. Weiterhin ist heutzutage unstreitig, dass in den Gesellschaftsstrukturen vieler Länder der Erde die Notwendigkeit und Üblichkeit zumindest von ,petty corruption“ so tief verwurzelt, und wenn nicht als probates Mittel gesellschaftlich anerkannt, dann zumindest so massenhaft akzeptiert ist, dass das geschriebene nationale Strafrecht wenig mehr als symbolhaften Charakter besitzt. Selbst wenn man davon ausgehen würde, dass die jeweiligen Strafverfolgungsbehörden in korruptionsanfälligen Staaten anders als die restliche Administration korruptionsfrei agieren würden, ${ }^{33}$ könnte dann positives Recht aufgrund der weitverbreiteten „Sozialadäquanz" seine Wirkung gleichwohl meist nur auf dem Papier entfalten. ${ }^{34}$ Randt ${ }^{35}$ hat dies so ausgedrückt: „Die Gepflogenheiten und Bräuche auf und auch innerhalb der verschiedenen kontinentalen Märkte unterscheiden sich z.T. erheblich voneinander, weswegen ein gewisser Mindeststandard, der wiederum Grundlage für einen strafrechtlichen Schutz wäre, real nicht existent ist. Die Ursachen dafür wurzeln in den unterschiedlichen wirtschaftspolitischen Ausformungen der Staatengemeinschaft, die maßgeblichen Einfluss auf die dortige Wettbewerbssituation haben, was sich wiederum auf die globale Wettbewerbssituation auswirkt“. Zumindest kann wohl nicht davon ausgegangen werden, dass überall auf der Welt gesellschaftliche und wirtschaftliche Wert-

32 Instruktiv beispielsweise eine Blacklist mit der üblichen Prozenthöhe des Bestechungsgeldes für verschiedenes Verwaltungs- und Justizhandeln in südamerikanischen Ländern bei Paul, Korruption in Deutschland und Brasilien; weiterführend zum tatsächlichen Befund Behr, in: FS Offerhaus, S. 345, 349 ff.; Leucht, StBP 1997, S. 117.

33 Gerade das ist aber häufig nicht der Fall. In Russland z.B. gehören Rechtsschutzorgane wie Polizei, Gerichte und Staatsanwaltschaften zu den am meisten unterwanderten Institutionen, siehe dazu Umfrageergebnisse der Stiftung Öffentliche Meinung (FOM) vom Dezember 2005 unter http://bd.fom.ru/report/map/projects/dominant/dom0601/ dom0601_4/tb0600106 sowie http://bd.fom.ru/report/map/projects/dominant/dom 0601/dom0601_4/tb06001067.

34 Ebenso Piehl, Bestechungsgelder im internationalen Wirtschaftsverkehr (1990), S. 64 ff., der in diesem Zusammenhang von „Papiertigern“ spricht. Rönnau in Achenbach/Ransiek, Handbuch Wirtschaftsstrafrecht, S. 111 geht (zum Parallelproblem bei § 299 Abs. 3 StGB) deswegen von Wettbewerbsnachteilen für deutsche Unternehmen gegenüber „einheimischen Konkurrenten" aus, weil die deutschen Strafverfolger im Gegensatz zu den einheimischen Behörden häufig tatsächlich ermitteln werden. Hinzuweisen verbleibt aber darauf, dass eine akzeptierte „Sozialadäquanz“ die Anwendung deutschen Strafrechts nicht zu hindern vermag.

35 Randt, BB 2002, S. 2252, 2256. 
maßstäbe - oder besser: Vorstellungen über den lauteren Wettbewerb - bestehen, die mit denen in der Bundesrepublik vergleichbar sind. In der Staatengemeinschaft existieren weltweit verschiedenste Staats-, Wirtschafts-, Steuer- und Sozialsysteme, welche unterschiedlichen Einfluss auf die Korruptionsanfälligkeit der Verwaltung und damit auch auf unternehmerische Chancen und unternehmerisches Handeln haben. Bedeutung gewinnt dieser Umstand, weil das deutsche Strafrecht gerade auch den Markt in denjenigen Ländern schützen will, die die OECD-Konvention gar nicht unterzeichnet haben, und sich dabei nicht etwa auf das Territorium der OECD-Mitgliedsstaaten beschränkt. ${ }^{36}$ Wenn nur 37 der weltweit ca. 190 Staaten diese Konvention unterzeichnet haben, ${ }^{37}$ rechtfertigt dies nicht zwingend die Annahme eines Konsenses dahingehend, dass der Weltmarkt gerade mit strafrechtlichen Mitteln zu schützen sei.

Vor diesem Hintergrund ist auch fraglich, ob alle Staaten, selbst wenn sie der Strafverfolgung bei grenzüberschreitender Korruption der eigenen Staatsbürger zugestimmt haben, bereit sind, diese tatsächlich in die Praxis umzusetzen - und zwar in einem mit deutschen Ermittlungsbehörden vergleichbaren Maß - wenn sie solcherlei Ermittlungen nicht für opportun halten. Eine OECD-Studie aus dem Jahr 2007 hat jedenfalls ergeben, dass in mehr als der Hälfte aller OECD-Unterzeichnerstaaten überhaupt keine Strafverfolgung bzgl. Auslandskorruption stattfindet. Dies gilt vor allem in exportstarken Nationen wie Großbritannien, Japan oder Kanada. Deutschland belegt dagegen bei der Anzahl der Ermittlungsverfahren wegen Auslandskorruption den zweiten Platz weltweit nach den USA mit 83 Verfahren bis zum Jahr 2007. ${ }^{38}$

Aufgrund der oben angesprochenen Divergenzen auf internationaler Ebene scheint daher ein internationales Rechtsgut „Lauterkeit des globalen Marktes“ zumindest im außereuropäischen Raum faktisch nicht vorhanden zu sein. ${ }^{39}$ Zumindest aber ist zweifelhaft, ob es tatsächlich ein gemeinsames, gleich stark ausgeprägtes Interesse der Staatengemeinschaft an der Reinheit des Marktes gibt. ${ }^{40}$

\section{Ursachen für Korruption und Ansätze für deren Bekämpfung}

Zwar fehlt es bis heute an einem allgemein anerkannten, vor allem aber empirisch verifizierbaren Erklärungsmodell für die Entstehung von Korruption. Von herausragender Bedeutung scheint nach dem Stand der Wissenschaft aber vor allem eine Überbürokratisierung von Verwaltungsabläufen zu sein, welche oft mit dem Anwachsen rechtlicher Regelungen einhergeht und von den Bürgern als „Normenflut“" oder „Normendickicht“ angesehen wird. ${ }^{41}$ Wenn die gesetzlichen Grundlagen zusätzlich noch kompliziert, unbestimmt (z.B. wegen übergroßen Ermessensspielräumen oder Regelungsdefiziten) und daher nicht ohne Weiteres einschätzbar sind, werden bei der Bevölkerung Anreize ge-

36 In diese Richtung gehend ebenso Zieschang, NJW 1999, S. 105, 107; Tinkl, wistra 2006, S. 126, 131.

37 Bannenberg, Korruption in Deutschland und ihre strafrechtliche Kontrolle (2002), S. 38.

38 Vgl. http://www.compliancemagazin.de/markt/hintergrund/transparency 190707.html.

39 So auch Randt, BB 2002, S. 2252, 2256; Steuerberater-Jahrbuch 2001/2002-Schaumburg, S. 239, 253 .

40 Ebenso Tinkl, wistra 2006, S. 126, 131.

41 Dazu eingehend Benz/Seibel-Wewer, Zwischen Kooperation und Korruption(1992), S. $306 \mathrm{ff}$. 
schaffen, das gewünschte Ergebnis auf informellem Wege schneller, sicherer und effizienter zu erreichen, insbesondere dann, wenn auch bei normgemäßem Verhalten und Erfüllen aller Voraussetzungen das Ergebnis des Verwaltungsverfahrens - auch zeitlich - nicht vorhersagbar ist. Einig ist man sich jedenfalls darüber, dass wahrscheinlich eine Vielzahl von Faktoren auf mehreren Ebenen korruptive Handlungen erheblich begünstigen und das Entstehungsrisiko von Korruption deutlich vergrößern kann..$^{42}$

Bei der Korruptionsbekämpfung stehen heutzutage jedoch längst nicht mehr nur strafrechtliche Mittel zur Verfügung. Ausgehend von der Erkenntnis, dass Korruption durch Missstände auf mehreren Ebenen (gesellschaftliche/institutionelle/individuelle Ebene) begünstigt wird, können Ansätze erwogen werden, die auf den verschiedenen Ebenen gegen die Entstehungsgründe von Korruption ineinandergreifend zum Tragen kämen.

Hierfür können strafrechtliche Mittel, aber auch außerstrafrechtliche Rechtsetzung sowie nichtlegislatorische Maßnahmen zur Korruptionsprävention eingesetzt werden. Interessant ist, dass sich insbesondere in den letzten Jahren in besonderem Maße Alternativen zur Strafrechtssetzung (und diesbezügliche neue Denkansätze) herausgebildet haben, die es früher in dieser Form nicht gab. Neuere Studien zeigen, dass sich diese außerstrafrechtlichen Mittel als praxistauglich und als mehrheitlich hocheffektiv zur Verhinderung von Korruption herausgestellt haben. Dies gilt beispielsweise für die Einrichtung einer korruptionsfeindlichen Institutionen- und Unternehmensstruktur, für die Implementierung einer schlagkräftigen Compliance-Organisation in Unternehmen, für die Installation von Ombudsleuten und Whistleblower-Hotlines in Unternehmen und Behörden, für die nachdrückliche Einforderung von Transparenz und Bürgernähe, für den ernstgemeinten Bürokratieabbau, aber beispielsweise auch für die Einrichtung von Korruptionsregistern (Black Lists). ${ }^{43}$

Das Vorhandensein dieser außerstrafrechtlichen Mittel, welche sich nachgewiesenermaßen als sehr effektiv in der Praxis der Korruptionsprävention bewiesen haben, führt deswegen vor dem Hintergrund des Ultima-Ratio-Prinzips zu der Frage, welchen Stellenwert das Strafrecht bei der Korruptionsbekämpfung im Verhältnis zu anderen Mitteln einnehmen soll. Mittlerweile ist in der Literatur die Ansicht vorherrschend, dass bei der Korruptionsbekämpfung Strafrecht zwar eine Rolle zu spielen habe, den Schwerpunkt solle aber eindeutig die Prävention bilden. ${ }^{44}$ Gerade im Bereich der Korruptionsdelinquenz dürfte eine situative Kriminalprävention ${ }^{45}$ besonders erfolgsversprechend sein, um den Besonderheiten des wirtschaftsnahen Tatumfelds möglichst gut gerecht zu werden. Beinahe einhellig wird weiterhin im Schrifttum betont, dass Strafrecht alleine kein taugliches Mittel zur Korruptionsbekämpfung darstellen könne. Das Strafrecht als

42 Zusammenfassung bei Claussen/Ostendorf, Korruption im öffentlichen Dienst (2002), S. $18 \mathrm{ff}$.

43 Vgl. etwa Wiehen in Pieth/Eigen, Korruption im internationalen Geschäftsverkehr, S. 492, $510 \mathrm{ff}$., der in diesem Zusammenhang von Korruptionsregistern als vom „bei weitem wirksamsten Abschreckungsmittel" spricht, weil den Unternehmen immense Vermögens- und Reputationseinbußen drohen können; vgl. weiterhin Ostendorf, NJW 1999, S. 615, 617.

44 Siehe statt vieler Ostendorf, NJW 1999, S. 615, 616; Möllering, WRP 1997, S. 933 ff.; Kerner/ Rixen, GA 1996, S. 355, 394; Vahlenkamp/Knau $\beta$, Korruption - hinnehmen oder handeln? Ein unscharfes Phänomen als Gegenstand zielgerichteter Prävention (1995), S. 250, 348 ff.

45 Frank/Harrer-Poerting, Der Sachverständige im Strafrecht (1990), S. 112 ff. 
das einschneidendste Instrumentarium, das sich schon oft als zu scharfes und zu viel zerstörendes Werkzeug erwiesen hat, darf nur als Ultima Ratio in Betracht kommen. ${ }^{46}$ Erst, wenn andere Mittel nicht verfangen, ist der Staat legitimiert, die scharfe Waffe des Strafrechts einzusetzen, um ein gewünschtes soziales Verhalten zu erzwingen; im Zweifel hat die Freiheit Vorrang.

Diese als Grundsatz ständig wiederholte (und manchmal gleichwohl vergessene) Ansicht scheint indes wenig berücksichtigt zu werden - auch im Bereich der Bekämpfung von Korruption auf ausländischen Märkten.

Zunächst kann das Nichtvorhandensein direkter Einflussmöglichkeiten mit Präventionswirkung bei der Verhinderung von Korruption auf Auslandsmärkten nicht dazu führen, dass bei der internationalen Korruptionsbekämpfung das Strafrecht deswegen nicht mehr als Ultima, sondern als Prima oder gar Sola Ratio angesehen wird. Der Gesetzgeber hat trotz aller Schwierigkeiten stattdessen indirekte Möglichkeiten zur Prävention bei Auslandssachverhalten als Alternative zum Einsatz des Strafrechtes zu erörtern. Evaluiert man den Kampf gegen internationale Korruption auf den Weltmärkten daraufhin, inwiefern auch außerstrafrechtliche Mittel - legislatorische und nicht-legislatorische - in der Praxis eingesetzt werden, lässt sich zunächst feststellen, dass die Bundesrepublik durchaus auch außerstrafrechtliche Mittel bei der grenzüberschreitenden Korruptionsbekämpfung anwendet. Demgegenüber hat sich der deutsche Gesetzgeber jedoch vorrangig dazu entschieden, die Repressionsmittel massiv auszuweiten. Das Strafbarkeitsrisiko für im Ausland tätige Unternehmen bzw. deren Mitarbeiter ist erheblich gestiegen, was in vielen Staaten zu einer Dilemmasituation führen dürfte. ${ }^{47}$

Die Wahl des Strafrechts als vorrangiges Mittel, um die Lauterkeit des internationalen Wettbewerbs zu schützen, ist vor allen Dingen auch deswegen bemerkenswert, da nach wie vor hochumstritten ist, ob und inwiefern das Strafrecht - zumal zusätzlich noch auf internationaler Ebene - Korruption überhaupt verhindern kann. Da korruptem Verhalten in Gesellschaft, Staat und Wirtschaft durch strikt realisierte Präventionsmaßnahmen nichtstrafrechtlicher Art erwiesenermaßen effektiv entgegengewirkt werden kann, bietet sich Korruption demnach nicht primär als Regelungsmaterie des Strafrechts an. Vor allem das Einfordern von Verwaltungsreformen zur Schaffung bzw. Erhöhung von Transparenz und Bürgernähe kann, wenn die Reformen tatsächlich implementiert werden, als in hohem Maße geeignet angesehen werden, die Korruptionsgefahr wirksam einzugrenzen. ${ }^{48}$ Die Bundesrepublik kann an dieser Stelle effektiv außerhalb des Strafrechts unterstützend tätig werden, etwa indem die Zusage von Entwicklungshilfe weit stärker als bisher an die Zusage des Empfängerlandes gebunden wird, entsprechende Reformen (Transparenz, Bürgernähe, Bürokratieabbau, Einführung von Korruptionsregistern usw.) umzusetzen und den Kampf gegen Korruption von innen heraus auch in der Praxis - und nicht nur auf dem Papier - aufzunehmen.

Da das Strafrecht kein primäres Mittel zur Verhaltenserzwingung sein kann, beseitigen Strafandrohungen aus Deutschland auch nicht den „Markt“ für korrupte Vorgehensweisen, erst recht nicht, wenn sich dieser „Markt“ im Ausland befindet. Wenn durch

46 Dieser Programmsatz ist an sich unstreitig, siehe BVerfGE 39, 1, 47; 57, 250, 270; 88, $203=$ NJW 1993, S. 1751, 1754; Baumann/Weber/Mitsch, Strafrecht AT, § 3 Rn. 19; Roxin, Strafrecht AT Bd. I § 2 Rn. 38 f.; Volk JZ 1982, S. 85, 88.

47 Vgl. dazu auch Dölling Handbuch Korruptionsprävention-Lembeck, S. 240, 288.

48 Ebenso Kerner/Rixen, GA 1996, S. 355, 394. 
die Kriminologie aber bereits für die Lage innerhalb der Bundesrepublik festgestellt wurde, dass die Präventionseffektivität des gesamten Strafrechtsarsenals im Bereich der Wirtschaftsdelinquenz kaum vorhanden zu sein scheint, ${ }^{49}$ fragt sich dann, wieso dieser Befund bei der Bekämpfung der internationalen Korruption auf den Märkten fremder Länder anders ausfallen sollte. Vielmehr wird man hier noch von weiteren Effektivitätseinbußen bei der Prävention ausgehen müssen. Grundsätzlich muss es daher Aufgabe der Nehmerländer sein, für die Sauberkeit ihrer Verwaltung und ihres Wirtschaftskreislaufs zu sorgen. ${ }^{50}$ Wo dies nicht gelingt, kann das deutsche Strafrecht nicht ohne Weiteres als gleichwertiger Ersatz herangezogen werden. ${ }^{51}$

\section{Erfolgsaussichten der weltweiten Korruptionsbekämpfung durch deutsches Strafrecht}

Trotz der unbestreitbaren Rolle, die Strafrecht innerhalb eines ganzheitlichen Ansatzes der Korruptionsbekämpfung zu spielen vermag, und trotz der Erfolge, die eine Kriminalisierung entsprechender Taten im Ausland für die Korruptionsprävention mit sich bringen kann, fragt sich dann, ob das Strafrecht die an es gestellten Anforderungen, also hier die Verhinderung von Korruption in allen Ländern weltweit, überhaupt bewältigen kann. Trotz einer Anzahl bereits verurteilter Personen, z.B. im Zuge des sog. ,,SiemensSkandals“, ist dann die Gefahr eines geradezu klassischen ,symbolischen Strafrechts ${ }^{\text {"52 }}$ gegeben, ${ }^{53}$ also ein Strafrecht als Mittel der Kriminalpolitik, das real nicht in der Lage ist, Rechtsgüterschutz zu gewährleisten, aber als ,,politisch“ deutlich sichtbarer Indikator der Handlungsfähigkeit des Staates wahrgenommen wird. Albrecht spricht in diesem Zusammenhang auch vom Strafrecht als ,politische Scheinwaffe“, 54 eine Formulierung, die für die deutsche Pönalisierung von Auslandskorruption in geradezu klassischer Weise zuzutreffen scheint. Zwar kann es u.U. angezeigt sein, die Symbolik einer Strafnorm nicht in ihrer Gänze abzulehnen, weil allein schon die Strafandrohung an sich ansatzweise lenkende Wirkung entfalten kann. ${ }^{55}$ Das Strafrecht verstünde sich aber dann trotzdem vor allem in Konstellationen mit hohem politischen „Problemlösungsbedarf"56 hauptsächlich als flexible „Waffe“, welche versucht, politische Wün-

49 Hefendehl, NJ 2006, S. 17.

50 Möllering, WRP 1997, S. 933 ff.; in diese Richtung gehend auch Prittwitz, in: Prittwitz/ Manoledakis, Strafrechtsprobleme an der Jahrtausendwende, S. 163, 172.

51 Ebenso Weigend, in: FS Jakobs, S. 747, 762.

52 Zum Begriff siehe NK-Hassemer/Neumann, vor § 1 Rn. 345; Hassemer, NStZ 1989, S. 553; ders., ZRP 1992, S. 378.

53 Wie hier Klaus Bernsmann unter www.lex.unicit.it/congressoitalotedesco/Bernsmann $\% 20$ (ted.).pdf., S. 12.

54 Albrecht, in: Institut für Kriminalwissenschaften Frankfurt am Main (Hrsg.), (1995), Vom unmöglichen Zustand des Strafrechts, S. 429.

55 Achenbach, GA 2004, S. 559, 565, sieht die Symbolik teilweise als legitimes Mittel ,politischen Marketings".

56 Insbesondere zu diesem Problem siehe Albrecht, in: Vom unmöglichen Zustand des Strafrechts, S. $429 \mathrm{ff}$. 
sche nach Problemlösungen zu bedienen: ${ }^{57}$ In der Tat ist der Ruf nach einem immer flexibleren Strafrecht in Medien und Gesellschaft, vor allem aber in der Politik allgegenwärtig. ${ }^{58}$ Gegen Missstände soll schnell, effektiv und sichtbar vorgegangen werden. Teilweise wird deswegen von einer Instrumentalisierung des Strafrechts durch Politik, Kultur und Massenmedien gesprochen, ${ }^{59}$ besonders bei spektakulären „Skandalen“, die die Öffentlichkeit in besonderem Maß beschäftigen.

Vielmehr dürfte es demgegenüber darauf ankommen, den „Markt“ für korrupte Vorgehensweisen möglichst weit zurückzudrängen. Eine Kriminalisierung allein hilft insofern nicht, weil es für die Verhinderung von Kriminalität auch entscheidend darauf ankommt, dass der Normadressat eine akzeptable, normkonforme Verhaltensalternative vorfindet. ${ }^{60}$ Gerade dies ist vielerorts jedoch nicht gegeben. ${ }^{61}$ Für Gesellschaft und Wirtschaft, vor allem aber auch für die ,verwalteten“ Menschen vieler betroffener Staaten wird es also wichtig sein, den Zwang zu beseitigen, für eigentlich selbstverständliche, legitime Dienstleistungen der Administration illegitime Zuwendungen leisten zu müssen; der Einzelne muss sich sicher sein, dass sein Gesuch in angemessener Frist, mit voraussehbarem, also rechtlich nachvollziehbarem Ergebnis willkürfrei und sachgerecht bearbeitet wird. Würde die Verwaltung nach solchen bürgerfreundlichen Grundsätzen (good governance) funktionieren, gäbe es kaum noch einen „Markt“ für Korruption. ${ }^{62}$ Dies kann hauptsächlich durch Einforderung von Rechtssicherheit, strikter Gewaltenteilung, unabhängiger Justiz, vor allem aber durch Transparenz und Bürgernähe in allen Bereichen erreicht werden. ${ }^{63}$ Transparenz gepaart mit effektiver gerichtlicher Kontrolle ist Voraussetzung für eine kritische Begleitung von politischen, administrativen und justiziellen Entscheidungen auf allen Ebenen. Dies kann nur durch entsprechende Reformen auf allen Ebenen der Gesellschaft realisiert werden. Deutsches (also vom betroffenen Staat aus gesehen ,ausländisches“) Strafrecht kann dies nicht leisten, es ist gerade kein Werkzeug zum „Nation Building“ im weitesten Sinne und wird deswegen - anders als immer wieder angekündigt - gemessen an seiner tatsächlichen Effektivität im Kampf gegen Korruption im Ausland ein stumpfes Schwert bleiben. ${ }^{64}$

Albrecht hat in diesem Zusammenhang richtigerweise auf einen weiteren relevanten Aspekt hingewiesen: Soll postmodernes Risikostrafrecht heutzutage vermehrt „weltgesellschaftliche Wohlstandsdiskrepanzen“ und „,sozio-ökonomische Konfliktlagen“ bekämpfen (wie sie mitunter auch in gesteigerter Korruptionsanfälligkeit beispielsweise einiger Entwicklungsländer zum Ausdruck kommen), wird es nicht nur deutlich überfordert. Erreicht wird dadurch auch, dass der strafjuristische Zugriff als politischer Aus-

57 Ebenso NK-Hassemer/Neumann, Vor § 1 Rn. 344; Albrecht, StV 1994, S. 265 ff.: damit einher gehen dann auch Rufe nach Entformalisierungen der Strafrechtsmaterie.

58 Albrecht, StV 1994, S. 265.

59 Albrecht, in: Vom unmöglichen Zustand des Strafrechts S. 429, 436.

60 Killias, in: FS-Schneider, S. 239, 250.

61 Vgl. nur für Russland Gilinskij, MSchrKrim 2007 (Heft 6), S. 502, 506 f.: „,.. Geben muss man für alles".

62 Ebenso Weigend, in: FS Jakobs, S. 747, 764 f.; siehe auch Horrer (o. Fn. 24), S. 300 f.

63 Für Russland siehe Gilinskij, MSchrKrim 2007 (Heft 6), S. 502, 510 ff.

64 Wie hier Weigend, in: FS Jakobs, S. 747, 762, 764 f.; Horrer (o. Fn. 24), S. 291 ff.; vgl. auch Bernsmann unter http://www.lex.unicit.it/congressoitalotedesco/Bernsmann\%20(ted.).pdf., S. 7. 
blendungs- und Verschleierungsmechanismus fungiert, ,durch den gesellschaftliche Probleme personalisiert werden, wodurch sie einer poltischen Zurechnung entgehen. Es wird ihnen ihre soziale Entstehungsgeschichte genommen und damit eine strukturpolitische Intervention erst gar nicht thematisiert. Die Auseinandersetzung wird systematisch auf Nebenschauplätze verlagert. Vom Kern des Problems wird abgelenkt“" ${ }^{65}$

Daher kann im Sinne Albrechts konstatiert werden, dass der deutsche Gesetzgeber im Bereich der grenzüberschreitenden Korruptionsprävention die Augen vor den tatsächlichen Gründen für die Korruptionsentstehung in anderen Ländern bewusst verschließt. Da ihm ein direkter Zugriff auf die gesellschaftliche, behördliche und rechtliche Ebene der anderen Staaten aus souveränitätsrechtlichen Gründen verwehrt ist, soll eben ein weltweit einsetzbares Strafrecht die Korruptionsanfälligkeit ausländischer Administrationen (bzw. „offiziell“ die Wettbewerbssituation in allen Ländern der Welt) ,in Ordnung bringen", ohne auf Souveränitätsbeschränkungen Rücksicht nehmen zu müssen. Die wahren Gründe der Korruptionsentstehung können damit jedoch nicht bekämpft werden. Das IntBestG verlangt von deutschen Unternehmen mehr Moral ab, als im täglichen Geschäftsleben vieler Länder ernsthaft zu erwarten ist; daher führt deutsches Recht nicht zu mehr Wettbewerbsgleichheit, sondern zu mehr Wettbewerbsungleichheit. Rund 150 Staaten haben das OECD-Abkommen nicht ratifiziert. Auch mit Unternehmen aus diesen Ländern stehen deutsche Unternehmen aber im Wettbewerb. Dass der Gesetzgeber die deutschen Unternehmen mit dieser Dilemmasituation, die in vielen Ländern faktisch vorherrscht, alleine lässt, erscheint unehrlich. Das deutsche Strafrecht wird zwar im Laufe der Zeit zahlreiche Verurteilungen deutscher Täter liefern, aber gemessen an seiner Zielsetzung scheitern. Es wird die an es gestellten Aufgaben nicht bewältigen können.

Fraglich ist, welche Rückschlüsse aus diesem Befund für die einschlägige Kriminalpolitik zu ziehen sind. Zu fordern ist hier namentlich ein deutlicher Vorrang von situativen Präventionsansätzen vor weiteren Repressionsbemühungen. Es ist zu bedauern, dass auf europäischer Ebene statt einer praktisch ineffizienten Kriminalisierung bis jetzt nicht auf andere - gerade in Bezug auf die Korruption - durchaus zur Verfügung stehende Präventionsmittel zurückgegriffen wurde. ${ }^{66}$ Einziger Lösungsweg: Den Markt für Korruption abschaffen helfen, akzeptable Verhaltensalternativen zur Verfügung stellen, also den Zwang der ,,verwalteten“ Menschen vor Ort abbauen helfen, dass auch für legitime Anliegen bezahlt werden muss. Hierfür kann die Bundesrepublik durchaus effektiv durch mittelbare Unterstützung des betroffenen Landes ihren Beitrag leisten mit außerstrafrechtlichen Mitteln.

65 Albrecht, in: Vom unmöglichen Zustand des Strafrechts, S. 429, 434: Hier wird das Problem unter dem Topos „Die Zurückweisung politischer Verantwortlichkeit durch die Individualisierung sozialer Problemlagen" behandelt.

66 Klaus Bernsmann unter www.lex.unicit.it/congressoitalotedesco/Bernsmann\%20(ted.).pdf, S. 12. 